\title{
AN UNCOUNTABLE COLLECTION OF MUTUALLY INCOMPARABLE CHAINABLE CONTINUA
}

\author{
MARWAN M. AWARTANI
}

(Communicated by James E. West)

\begin{abstract}
We exhibit the existence of continuum many compactifications of the ray with the arc as remainder, no one of which maps onto any other. We also obtain continuum many pathwise connected circularly chainable continua no one of which maps onto any other. The involved constructions and proofs are combinatorial in nature.
\end{abstract}

\section{INTRODUCTION}

In [8] Rogers raised the question: "Does there exist an uncountable collection of chainable continua, no member of which maps onto any other?" In [3] Bellamy gave an affirmative answer to this question. However each member of Bellamy's collection consisted of infinitely many path components. The purpose of this paper is to prove the existence of the desired collection within the family of compactifications of the the ray with the arc as remainder, whose members consist of exactly two path components. This also generalizes the main result in [1]. For related work see [2, 6, 7].

As a corollary, we obtain the known result $[3,5,9]$ that there exists an uncountable collection of circularly chainable continua, no member of which maps onto any other. Unlike the continua in $[3,5,9]$, which consist of infinitely many path components, each member of this collection is pathwise connected.

First we define a partial order on the class of all sequences of zeroes and ones and prove that with respect to the given partial order, this class contains uncountably many mutually incomparable elements. Then we associate with each sequence of zeroes and ones a compactification of the ray with the arc as remainder. Finally we prove that if a surjective map exists between a pair of such compactifications then the underlying sequences are comparable.

Received by the editors March 17, 1990; presented at the 1989 Spring Topology Conference held in Knoxville, Tennessee.

1991 Mathematics Subject Classification. Primary 54D35, 54F20.

Key words and phrases. Chainable and circularly chainable continua, compactification, mapping between continua. 


\section{Preliminary Results}

$N$ denotes the set of natural numbers.

2.1. Definition. Let $2^{N}$ denote the set of all sequences of zeroes and ones, and let $\left\{a_{i}\right\},\left\{b_{i}\right\}$ be two elements in $2^{N}$. Then

(i) $\left\{a_{i}\right\}$ is said to vertically dominate $\left\{b_{i}\right\}$ if $a_{i} \geq b_{i}$ eventually.

(ii) $\left\{a_{i}\right\}$ is said to dominate $\left\{b_{i}\right\}$ if there exists an integer $j_{0}$ such that $a_{i} \geq b_{i+j_{0}}$ eventually.

(iii) $\left\{a_{i}\right\}$ and $\left\{b_{i}\right\}$ are called incomparable if neither of them dominates the other.

The following lemma is easy to verify.

2.2. Lemma. Let $\left\{a_{i}\right\},\left\{b_{i}\right\}$ be two elements in $2^{N}$. If $\left\{a_{i_{j}}\right\}_{j=1}^{\infty}$ is a tail of the sequence of zeroes in $\left\{a_{i}\right\}$, and if for some integer $j_{0}, b_{i_{j}+j_{0}}$ is eventually zero, then $\left\{a_{i}\right\}$ dominates $\left\{b_{i}\right\}$.

2.3. Lemma. $2^{N}$ contains continuum many elements no one of which vertically dominates any other.

Proof. Let $P(N)$ denote the power set of $N$. For each $\alpha=\left\{\alpha_{i}\right\} \in 2^{N}$ define $F(\alpha) \in P(N)$ as follows: $i \in F(\alpha)$ iff $\alpha_{i}=1$. $F$ is clearly a bijection. Moreover, if $F(\alpha)$ and $F(\beta)$ are infinite and almost disjoint (i.e., $F(\alpha) \cap F(\beta)$ is finite) then $\alpha$ and $\beta$ are vertically incomparable. The result now follows since it is well known that $P(N)$ contains a collection of cardinality $c$ consisting of mutually almost disjoint infinite subsets of $N$.

2.4. Lemma. $2^{N}$ contains continuum many elements none of which is eventually constant and no one of which dominates any other.

Proof. For each $\alpha=\left\{\alpha_{i}\right\} \in 2^{N}$ define a sequence $\left\{a_{n}^{\alpha}\right\}$ as follows:

$$
\begin{array}{ll}
a_{n}^{\alpha}=0, & n \neq 2^{j} \text { or } 2^{j}+1 ; \\
a_{n}^{\alpha}=1, & n=2^{j} ; \\
a_{n}^{\alpha}=\alpha_{j}, & n=2^{j}+1 .
\end{array}
$$

It is obvious that if $\left\{a_{n}^{\alpha}\right\}$ dominates $\left\{a_{n}^{\beta}\right\}$ then $\alpha$ vertically dominates $\beta$. The result now follows from Lemma 2.3.

\section{Constructing the unCOUNTABle COllection}

3.1. Notation. Throughout this paper, if $f:(0,1] \rightarrow[0,1]$ is a continuous function, then the points of the graph of $f$ are ordered by their first coordinate. Moreover if $a$ and $b$ are two points in the graph, then $(a, b),[a, b]$ denote respectively the open and closed portion of the graph between $a$ and $b$. If $A$ is a subarc of the graph, then $d(A)$ denotes the length of $A$ measured along the graph. $\Pi_{1}$ and $\Pi_{2}$ denote the standard projections on the first and second coordinates respectively. All maps are assumed to be continuous.

3.2. Construction. With each $\alpha=\left\{\alpha_{i}\right\} \in 2^{N}$, we associate a compactification $E_{\alpha}$ of the ray $J_{\alpha}$ with the arc $I_{\alpha}$ as remainder. It suffices to describe $J_{\alpha}$.

(i) $J_{\alpha}$ is the graph of a piecewise linear function from $(0,1]$ to $[0,1]$. 
(ii) The point $(1,1 / 2) \in J_{\alpha}$.

(iii) Let $m_{\alpha}$ and $M_{\alpha}$ denote respectively the set of minima and the set of maxima of $J_{\alpha}$ and let $V_{\alpha}=m_{\alpha} \cup M_{\alpha}$.

(a) If $\alpha_{i}=0$ then $\left[a_{i}, b_{i}\right] \cap V_{\alpha}$ has the following properties: (1) $\mid\left[a_{i}, b_{i}\right] \cap$ $M_{\alpha} \mid=2 i$ and $\left|\left[a_{i}, b_{i}\right] \cap m_{\alpha}\right|=2 i-1$. (2) If $\left\{M_{i, j}: 1 \leq j \leq 2 i\right\}$ is an enumeration from right to left of the elements of the set $\left[a_{i}, b_{i}\right] \cap M_{\alpha}$ then $\pi_{2}\left(M_{i, 2 i}\right)=1$ and $\pi_{2}\left(M_{i, j}\right)=\pi_{2}\left(M_{i, 2 i-j}\right)=(j+2) /(j+3) \forall j, 1 \leq j \leq i$. (3) $\pi_{2}\left(\left[a_{i}, b_{i}\right] \cap m_{\alpha}\right)=1 / 2$.

(b) If $\alpha_{i}=1$ then $\left[a_{i}, b_{i}\right] \cap V_{\alpha}$ has the following properties: (1) $\mid\left[a_{i}, b_{i}\right] \cap$ $M_{\alpha} \mid=2 i+1$ and $\left|\left[a_{i}, b_{i}\right] \cap m_{\alpha}\right|=2 i$. (2) If $\left\{M_{i, j}: 1 \leq j \leq 2 i+1\right\}$ is an enumeration from right to left of the elements of the set $\left[a_{i}, b_{i}\right] \cap M_{\alpha}$ then $\pi_{2}\left(M_{i, 2 i+1}\right)=1$ and $\pi_{2}\left(M_{i, j}\right)=\pi_{2}\left(M_{i, 2 i+1-j}\right)=(j+2) /(j+3) \forall j, 1 \leq j \leq i$. (3) If $\left\{m_{i, j}: 1 \leq j \leq 2 i\right\}$ is an enumeration from right to left of the elements of the set $\left[a_{i}, b_{i}\right] \cap m_{\alpha}$ then $\pi_{2}\left(m_{i, j}\right)=2 / 3$ for $j=i$ and $1 / 2$ for $j \neq i$.

(c) For each $i \in N,\left[b_{i}, a_{i}\right] \cap V_{\alpha}$ has the following properties: (1) $\mid\left[b_{i}, a_{i}\right] \cap$ $M_{\alpha} \mid=2 i-1$ and $\left|\left[b_{i}, a_{i}\right] \cap m_{\alpha}\right|=2 i$. (2) $\pi_{2}\left(\left[b_{i}, a_{i}\right] \cap M_{\alpha}\right)=1 / 2$. (3) If $\left\{m_{i, j}: 1 \leq j \leq 2 i\right\}$ is an enumeration from right to left of the elements of the set $\left[b_{i}, a_{i}\right] \cap m_{\alpha}$ then $\pi_{2}\left(m_{i, 2 i}\right)=0$ and $\pi_{2}\left(m_{i, j}\right)=\pi_{2}\left(m_{i, 2 i-j}\right)=$ $1-(j+2) /(j+3) \forall j, 1 \leq j \leq i$.

3.3. Notation. Unless mentioned otherwise, the following notations are used for the above-constructed compactification $E_{\alpha}$.

(i) Let $a_{\alpha}, b_{\beta}, c_{\alpha}$ denote respectively the points $(0,0),(0,1 / 2)$, and $(0,1)$ in $I_{\alpha}$.

(ii) For each $i \in N$ let $K_{i}^{\alpha}$ and $L_{i}^{\alpha}$ denote the arcs $\left[a_{i}, b_{i}\right]$ and $\left[b_{i}, a_{i+1}\right]$ respectively.

(iii) For each $i \in N$ let $u_{i}^{\alpha}$ denote the arc in $J_{\alpha}$ joining the last element of $L_{i}^{\alpha} \cap m_{\alpha}$ with the first element of $K_{i+1}^{\alpha} \cap M_{\alpha}$. Similarly, let $w_{i}^{\alpha}$ denote the arc in $J_{\alpha}$ joining the last element of $K_{i}^{\alpha} \cap M_{\alpha}$ with the first element of $L_{i}^{\alpha} \cap m_{\alpha}$. The following lemma is easy to verify.

3.4. Lemma. For a compactification $E_{\alpha}$ the following hold:

(i) If $t \in\left(a_{\alpha}, c_{\alpha}\right)$ and $t^{\prime} \in I_{\alpha}$ then there exist sequences $\left\{t_{i}\right\}$ and $\left\{t_{i}^{\prime}\right\}$ in $J_{\alpha}$ converging to $t$ and $t^{\prime}$ respectively such that $\left\{d\left[t_{i}, t_{i}^{\prime}\right]\right\}$ is bounded away from infinity.

(ii) Let $\left\{t_{i}\right\}$ and $\left\{t_{i}^{\prime}\right\}$ be two sequences in $J_{\alpha}$ converging to $a_{\alpha}$ and $c_{\alpha}$ respectively; then $\lim d\left[t_{i}, t_{i}^{\prime}\right]=\infty$.

(iii) For each $i \in N$ let $p_{i} \in L_{i}^{\alpha}$; then $\lim d\left[p_{i}, p_{i+1}\right]=\infty$.

(iv) Let $\left\{p_{i}\right\}$ and $\left\{q_{i}\right\}$ be two sequences in $J_{\alpha}$ both converging to $a_{\alpha}$. If $\left\{d\left[p_{i}, q_{i}\right]\right\}$ is bounded away from zero then $\left[a_{\alpha}, b_{\alpha}\right] \subseteq \lim \left[p_{i}, q_{i}\right]$.

(v) Let $\left\{A_{i}\right\}$ be a sequence of arcs in $J_{\alpha}$ such that $A_{i} \cap w_{j_{i}}^{\alpha} \neq \varnothing$ eventually. If $\lim A_{i} \supset\left[a_{\alpha}, b_{\alpha}\right]$ then $\lim d\left(A_{i}\right)=\infty$.

(vi) Let $p \in u_{k_{i}}^{\alpha}$ and $q \in w_{k_{i}+1}^{\alpha}$, then $[p, q] \cap m_{\alpha} \cap(y=2 / 3)=\varnothing$ iff $\alpha_{k_{i}}=0$.

\section{The Main Results}

4.1. Lemma. Let $h: E_{\alpha} \rightarrow E_{\beta}$ be a surjective map, and let $A$ be a closed arc in $J_{\alpha}$. If $B$ is a closed arc in $h(A)$ then there exists a closed arc in $A$ whose image is $B$. 
Proof. The result follows directly since the map $h \mid A: A \rightarrow h(A)$ is simply a map between closed intervals.

4.2. Lemma. Let $h: E_{\alpha} \rightarrow E_{\beta}$ be a surjective map, and let $\left\{A_{i}\right\}$ be a sequence of arcs in $J_{\alpha}$ such that $\limsup A_{i} \subseteq I_{\alpha}$. The following hold.

(i) If $\left\{d\left(h\left(A_{i}\right)\right)\right\}$ is bounded away from zero then $\left\{d\left(A_{i}\right)\right\}$ is bounded away from zero.

(ii) If $\left\{d\left(A_{i}\right)\right\}$ is bounded away from infinity then $\left\{d\left(h\left(A_{i}\right)\right)\right\}$ is bounded away from infinity.

Proof. (i) We may assume without loss of generality that $\left\{A_{i}\right\}$ is a convergent sequence. Since $\left\{d\left(h\left(A_{i}\right)\right)\right\}$ is bounded away from zero, each $h\left(A_{i}\right)$ contains a pair of points $p_{i}, q_{i}$ such that $\lim p_{i} \neq \lim q_{i}$. Hence $A_{i}$ contains a pair of points $p_{i}^{\prime}, q_{i}^{\prime}$ such that $\lim p_{i}^{\prime} \neq \lim q_{i}^{\prime}$, which implies that $\left\{d\left(A_{i}\right)\right\}$ is bounded away from zero.

(ii) Suppose that $\lim d\left(h\left(A_{i}\right)\right)=\infty$. Then for each $i \in N, h\left(A_{i}\right)$ contains a collection $\left\{B_{i, j}: 1 \leq j \leq i\right\}$ of disjoint closed subarcs such that $\left\{d\left(B_{i, j}\right):(i, j) \in N \times\{1,2, \ldots, i\}\right\}$ is bounded away from zero. For each $i \in N$ and each $j, 1 \leq j \leq i$, let $A_{i, j}$ be a subarc of $A_{i}$ such that $h\left(A_{i, j}\right)=$ $B_{i, j}$. This is possible by Lemma 4.1. It follows from part (i) that $\left\{d\left(A_{i, j}\right)\right.$ : $(i, j) \in N \times\{1, \ldots, i)\}$ is bounded away from zero, implying that $\lim d\left(A_{i}\right)=$ $\infty$.

4.3. Lemma. Let $h: E_{\alpha} \rightarrow E_{\beta}$ be a surjective map. Then the following hold.

(i) $h^{-1}\left\{a_{\beta}, c_{\beta}\right\}=\left\{a_{\alpha}, c \alpha\right\}$.

(ii) If $h\left(a_{\alpha}\right)=a_{\beta}$, then $h\left[a_{\alpha}, b_{\alpha}\right]=\left[a_{\beta}, b_{\beta}\right]$ and $h\left[b_{\alpha}, c_{\alpha}\right]=\left[b_{\beta}, c_{\beta}\right]$.

(iii) If $h\left(a_{\alpha}\right)=c_{\beta}$, then $h\left[a_{\alpha}, b_{\beta}\right]=\left[c_{\beta}, b_{\beta}\right]$ and $h\left[b_{\alpha}, c_{\alpha}\right]=\left[b_{\beta}, a_{\beta}\right]$.

(iv) $h\left(b_{\alpha}\right)=b_{\beta}$.

Proof. (i) It suffices to prove that $\left(a_{\alpha}, c_{\alpha}\right) \cap h^{-1}\left(c_{\beta}\right)=\varnothing=\left(a_{\alpha}, c_{\alpha}\right) \cap h^{-1}\left(a_{\beta}\right)$. Suppose that $\left(a_{\alpha}, c_{\alpha}\right) \cap h^{-1}\left(a_{\beta}\right) \neq \varnothing$, and let $t^{\prime} \in\left[a_{\alpha}, c_{\alpha}\right], t \in\left(a_{\alpha}, c_{\alpha}\right)$, be chosen so that $h\left(t^{\prime}\right)=c_{\beta}$ and $h(t)=a_{\beta}$. By Lemma 3.4(i) we can find sequences $\left\{t_{i}\right\}$ and $\left\{t_{i}^{\prime}\right\}$ in $J_{\alpha}$ such that $\lim t_{i}=t$ and $\lim t_{i}^{\prime}=t^{\prime}$ where $\left\{d\left[t_{i}, t_{i}^{\prime}\right]\right\}$ is bounded away from infinity. Since $\lim h\left(t_{i}\right)=a_{\beta}$ and $\lim h\left(t_{i}^{\prime}\right)=c_{\beta}$, it follows from Lemma 3.4(ii) that $\lim d\left[h\left(t_{i}\right), h\left(t_{i}^{\prime}\right)\right]=\infty$. This contradicts Lemma 4.2(ii). It can be similarly shown that $h^{-1}\left(c_{\beta}\right) \cap\left(a_{\alpha}, c_{\alpha}\right)=\varnothing$.

(ii) We only prove that $h\left[b_{\alpha}, c_{\alpha}\right]=\left[b_{\beta}, c_{\beta}\right]$ since the proof that $h\left[a_{\alpha}, b_{\alpha}\right]=$ $\left[a_{\beta}, b_{\beta}\right]$ is similar and thus omitted. Since $h\left(a_{\alpha}\right)=a_{\beta}$, (i) implies that $h^{-1}\left(c_{\beta}\right)=c_{\alpha}$. Suppose that there exists $t \in\left(b_{\alpha}, c_{\alpha}\right)$ such that $h(t)<b_{\beta}$. Let $T$ denote the sequence $J_{\alpha} \cap(y=t)$. Two cases arise:

Case (a). There exist two subsequences $\left(p_{i}\right)$ and $\left(q_{i}\right)$ of $T$ such that for each $i \in N$ : (1) $p_{i}$ and $q_{i}$ are adjacent in $T$, (2) $\left\{p_{i}, q_{i}\right\} \subseteq K_{j_{i}}^{\alpha}$, and (3) $h\left(p_{i}\right) \in L_{k_{i}}^{\beta}$ and $h\left(q_{i}\right) \in L_{r_{i}}^{\beta}$ with $r_{i} \neq k_{i}$. It follows from Lemma 3.4(iii) that $\lim d\left[h\left(p_{i}\right), h\left(q_{i}\right)\right]=\infty$ and consequently that $\lim \left(h\left[p_{i}, q_{i}\right]\right)=\infty$. This contradicts Lemma 4.2 (ii) since $\left\{d\left[p_{i}, q_{i}\right]\right\}$ is bounded away from infinity.

Case (b). $h\left(K_{i}^{\alpha} \cap T\right) \subseteq L_{j_{i}}^{\beta} \forall i>i_{0}$. Then there exists a subsequence $\left\{K_{r}^{\alpha}\right\}_{i=1}^{\infty}$ such that $h\left(K_{r_{i}}^{\alpha} \cap T\right) \subseteq L_{j_{i}}^{\beta}$ where $j_{i} \neq j_{k}$ whenever $i \neq k$. For each $i \in N$ let $p_{r_{i}}, q_{r_{i}}$ be the first and last points respectively of $k_{r_{i}}^{\alpha} \cap T$. Since $L_{j_{i}}^{\beta} \cap L_{j_{i+1}}=\varnothing$, 
it follows that $h\left[q_{r_{i}}, p_{r_{i+1}}\right] \supset K_{l_{i}}^{\beta}$ for some $l_{i} \in N$, which in turn implies that $h\left(\lim \left[q_{r_{i}}, p_{r_{i+1}}\right]\right) \supset \lim K_{l_{i}}^{\beta}$. That is, $c_{\beta} \in h\left[a_{\alpha}, t\right]$ with $t<c_{\alpha}$, contradicting our assumption that $h^{-1}\left(c_{\beta}\right)=c_{\alpha}$. The proof of (iii) is similar and thus omitted and the proof of (iv) follows directly from (ii) and (iii) above.

4.4. Lemma. Let $h: E_{\alpha} \rightarrow E_{\beta}$ be a surjective map. If neither $\alpha$ nor $\beta$ is eventually constant then the following hold.

(i) $h\left(a_{\alpha}\right)=a_{\beta} ; h\left(c_{\alpha}\right)=c_{\beta} ; h\left[a_{\alpha}, b_{\alpha}\right]=\left[a_{\beta}, b_{\beta}\right] ; h\left[b_{\alpha}, c_{\alpha}\right]=\left[b_{\beta}, c_{\beta}\right]$.

(ii) If $\left\{A_{i}\right\}$ is a sequence of arcs in $J_{\alpha}$ such that $\lim A_{i}=\left[b_{\alpha}, c_{\alpha}\right]$ and $A_{i} \cap m_{\alpha} \cap(y=2 / 3)=\varnothing, \forall i \in N$, then $h\left(A_{i}\right) \cap m_{\beta} \cap(y=2 / 3)=\varnothing$ eventually.

Proof. (i) By Lemma 4.3(i), it suffices to prove that $h\left(a_{\alpha}\right)=a_{\beta}$. Suppose that $h\left(a_{\alpha}\right)=c_{\beta}$. It follows from Construction 3.2 that there exist sequences $\left\{p_{i}\right\}$ and $\left\{q_{i}\right\}$ in $J_{\beta}$ such that $\lim p_{i}=\lim q_{i}=c_{\beta}, \lim \left[p_{i}, q_{i}\right]=\left[2 / 3, c_{\beta}\right]$, and $\left\{d\left[p_{i}, q_{i}\right]\right\}$ is bounded away from zero. By Lemma 4.1 we can find a sequence $\left\{\boldsymbol{B}_{i}\right\}$ of arcs in $J_{\alpha}$ such that $h\left(B_{i}\right)=\left[p_{i}, q_{i}\right]$. Since $\left\{d\left[p_{i}, q_{i}\right]\right\}$ is bounded away from zero, it follows from Lemma 4.2(i) that $\left\{d\left(B_{i}\right)\right\}$ is bounded away from zero. Choose $p_{i}$ and $q_{i}$ in $B_{i}$ such that $h\left(p_{i}\right)=p_{i}$ and $h\left(q_{i}\right)=q_{i}$. Since $h^{-1}\left(c_{\beta}\right)=a_{\alpha}$, it follows that $\lim p_{i}=\lim q_{i}=a_{\alpha}$. We may assume without loss of generality that $\left\{B_{i}\right\}$ is a convergent sequence. Hence by Lemma 3.4(iv), $\left[a_{\alpha}, b_{\alpha}\right] \subseteq \lim B_{i}$. Since $\lim h\left(B_{i}\right)=\left[2 / 3, c_{\beta}\right]$, it follows that $h\left[a_{\alpha}, b_{\alpha}\right] \supset$ $\left[2 / 3, c_{\beta}\right]$, contradicting Lemma 4.3(iv). Hence $h\left(c_{\alpha}\right)=c_{\beta}$ and $h\left(a_{\alpha}\right)=a_{\beta}$. The proof of (ii) is similar and thus omitted.

4.5. Lemma. Let $h: E_{\alpha} \rightarrow E_{\beta}$ be a surjective map, and let $\left\{p_{i}\right\}$ and $\left\{q_{i}\right\}$ be sequences of points in $J_{\alpha}$ such that for each $i \in N, p_{i} \in u_{i}^{\alpha}$ and $q_{i} \in w_{i}^{\alpha}$. If $\lim q_{i}$ and neither $\alpha$ nor $\beta$ is eventually constant, then there exists an integer $j_{0}$ such that eventually $h\left(p_{i}\right) \in u_{i+j_{0}}^{\beta}$ and $h\left(q_{i}\right) \in w_{i+j_{0}}^{\beta}$.

Proof. Since $h\left(b_{\alpha}\right)=b_{\beta}$, it follows that $\lim h\left(p_{i}\right)=\lim h\left(q_{i}\right)=b_{\beta}$. We break the proof into four steps:

Step 1. $h\left(p_{i}\right) \in \bigcup_{j \in N} u_{j}^{\beta}$ eventually. Suppose not, then three cases arise:

Case (i). $\left\{d\left[h\left(p_{i}\right), M_{j_{i}}\right]\right\}$ converges to zero for some subsequences $\left\{M_{j_{i}}\right\}_{i=1}^{\infty}$ of $M_{\beta}$. Then it can be shown using the uniform continuity of $h$ that $\exists \delta>$ $0, \ni h\left[b_{\alpha}, b_{\alpha}+\delta\right] \subseteq\left[a_{\beta}, b_{\beta}\right]$ contradicting Lemma 4.4(i).

Case (ii). $\left\{d\left[h\left(p_{i}\right), m_{j_{i}}\right]\right\}$ converges to zero for some subsequence $\left\{m_{j_{i}}\right\}$ of $m_{\beta}$. Using a reasoning similar to the above, it can be shown that $\exists \delta>0$, $\ni h\left[b_{\alpha}, b_{\alpha}-\delta\right] \subseteq\left[b_{\beta}, c_{\beta}\right]$ again contradicting Lemma 4.4(i).

Case (iii). $\quad h\left(p_{i}\right) \in \bigcup_{j \in N} w_{j}^{\beta}$ eventually. Let $m_{i}^{*}=u_{i}^{\alpha} \cap m_{\alpha}$. Since $\lim \left[m_{i}^{*}, p_{i}\right]=\left[a_{\alpha}, b_{\alpha}\right]$, Lemma 4.4 implies that

$$
\lim h\left[m_{i}^{*}, p_{i}\right]=\left[a_{\beta}, b_{\beta}\right] .
$$

Since $h\left[m_{i}^{*}, p_{i}\right] \cap\left(\bigcup_{j \in N} w_{j}^{\beta}\right) \neq \varnothing$ eventually, it follows from Lemma 3.4(v) that $\lim d\left(h\left[m_{i}^{*}, p_{i}\right]\right)=\infty$. This contradicts Lemma 4.2(ii) since $\left\{d\left[m_{i}^{*}, p_{i}\right]\right\}$ is bounded away from infinity. Hence the only remaining possibility is for $h\left(p_{i}\right)$ to be eventually in $\bigcup_{j \in N} u_{j}^{\beta}$.

Step 2. $h\left(q_{i}\right) \in \bigcup_{j \in N} w_{j}^{\beta}$ eventually. The proof is similar to that in Step 1 and thus omitted. 
Step 3. If $h\left(p_{i}\right) \in u_{j_{i}}^{\beta}$ eventually, then $h\left(q_{i}\right) \in w_{j_{i}}^{\beta}$ eventually. Suppose not, then $h\left(q_{i}\right) \in w_{k_{i}}^{\beta}$ where $k_{i} \neq j_{i}$ infinitely often. Then it can be deduced that $h\left[a,{ }_{\alpha}, b_{\alpha}\right] \cap\left(b_{\beta}, c_{\beta}\right) \neq \varnothing$ contradicting Lemma 4.4(i).

Step 4. We finally prove that if $h\left(p_{i}\right) \in u_{j_{i}}^{\beta}$ eventually then $j_{i+1}=j_{i}+$ 1. Suppose that $j_{i+1} \neq j_{i}+1$ infinitely often; then it can be deduced that $h\left[b_{\alpha}, c_{\alpha}\right] \cap\left(a_{\beta}, b_{\beta}\right) \neq \varnothing$ contradicting Lemma 4.4(i).

It follows from the above that there exists an integer $j_{0}$ such that $h\left(p_{i}\right) \in$ $u_{i+j_{0}}^{\beta}$ and $h\left(q_{i}\right) \in w_{i+1_{0}}^{\beta}$ eventually. This completes the proof of the lemma.

4.6. Theorem. Let $h: E_{\alpha} \rightarrow E_{\beta}$ be a surjective map. If neither $\alpha$ nor $\beta$ is eventually constant then $\alpha$ dominates $\beta$.

Proof. For each $i \in N$ choose $p_{i} \in w_{i}^{\alpha}$ and $q_{i} \in w_{i}^{\alpha}$ such that $\lim p_{i}=$ $\lim q_{i}=b_{\alpha}$. By Lemma 4.5, there exists an integer $j_{0}$ such that $h\left(p_{i}\right) \in u_{i+j_{0}}^{\beta}$ and $h\left(q_{i}\right) \in w_{i+j_{0}}^{\beta}$ eventually. Let $\left\{a_{k_{i}}\right\}$ be a tail of the sequence of all zeros in $\alpha$. By Lemma 2.2, it suffices to prove that $\beta_{k_{i}+j_{0}}=0$ eventually. Since $\alpha_{k_{i}}=0$, it follows from Lemma 3.4(vi) that $\left[p_{k_{i}}, q_{k_{i}}\right] \cap m_{\alpha} \cap(y=2 / 3)=\varnothing$. By Lemma 4.4(ii), $h\left[p_{k_{i}}, q_{k_{i}}\right] \cap m_{\beta} \cap(y=2 / 3)=\varnothing$ eventually and hence $\left[h\left(p_{k_{i}}\right), h\left(q_{k_{i}}\right)\right] \cap$ $(y=2 / 3)=\varnothing$ eventually. Since $h(p) \in u_{k_{i}+j_{0}}^{\beta}$ and $h\left(q_{k_{i}}\right) \in w_{k_{i}+j_{0}}^{\beta}$, we conclude by Lemma 3.4(vi) that $\beta_{k_{i}+j_{0}}=0$ evenually, which completes the proof of the theorem.

Combining Lemma 2.4 and Theorem 4.6, we readily obtain the main result.

4.7. Theorem. There exists an uncountable collection of cardinality $c$ of compactifications of the ray with the arc as remainder, no member of which maps onto any other.

4.8. Corollary. There exists an uncountable collection of planar pathwise connected circularly chainable continua, no member of which maps onto any other. Proof. For each $\alpha \in 2^{N}$ let $E_{\alpha}$ be the associated compactification of the ray described in Construction 3.2. From $E_{\alpha}$ we obtain a circularly chainable continuum $E_{\alpha}^{\prime}$ by simply trying the first point of $J_{\alpha}$ with the point $a_{\alpha} \in I_{\alpha} . E_{\alpha}^{\prime}$ is clearly pathwise connected and planar. Notice that $E_{\alpha}^{\prime}-\left(a_{\alpha}, c_{\alpha}\right]$ is a ray densely embedded in $E_{\alpha}$. If $h: E_{\alpha}^{\prime} \rightarrow E_{\beta}^{\prime}$ is a surjective map then it is easy to verify that there exists a subray $J_{\alpha}^{\prime}$ of $E_{\alpha}^{\prime}-\left(a_{\alpha}, c_{\alpha}\right]$ having the following properties:

(i) If $\bar{J}_{\alpha}^{\prime}$ denotes the closure of $J_{\alpha}^{\prime}$ in $E_{\alpha}^{\prime}$ then $\bar{J}_{\alpha}^{\prime}-J_{\alpha}^{\prime}=I_{\alpha}$.

(ii) $h\left(J_{\alpha}^{\prime}\right)=J_{\beta}$.

Consequently $h \mid \overline{J_{\alpha}^{\prime}}: \overline{J_{\alpha}^{\prime}} \rightarrow \overline{J_{\beta}}$ is a surjective map for which the preceding results in 4.1-4.6 still hold from Theorem 2.4.

\section{REFERENCES}

1. M. M. Awartani, Compactifications of the ray with the arc as remainder, Topology Proc. 9 (1984), 201-215.

2. __ The fixed remainder property for self homeomorphisms of Elsa continua, Topology Proc. 11 (1986), 225-238.

3. D. P. Bellamy, An uncountable collection of chainable continua, Trans. Amer. Math. Soc. 160 (1971), 297-304. 
4. R. H. Bing, Snake like continua, Duke Math. J. 18 (1951), 653-663. Mr 13, 265.

5. H. Cook, Upper semicontinuous continuum valued mappings onto circle-like continua, Fund. Math. 60 (1967), 233-239. Mr 36, 3321.

6. T. Maćkowiak, The condensation of singularities in arclike continua, Houston J. Math. 11 (1985), 535-558.

7. __ Singular arclike continua, Dissertations Math. (Rozprawy Mat.) 257 (1986), 1-35.

8. J. W. Rogers, Jr., Continuous mappings on continua, Proc. Auburn Topology Conference, March 1969, pp. 94-97.

9. Pseudo-circles and universal circularly chainable continua, Doctoral dissertation, Univ. of California, Riverside, CA, 1968.

Department of Mathematics, Birzeit University, Birzeit, West Bank 